\title{
Terahertz Frequency Continuous-Wave Spectroscopy and Imaging of Explosive Substances
}

\author{
Michael A. Startsev and Abdulhakem Y. Elezzabi \\ Ultrafast Optics and Nanophotonics Laboratory, Department of Electrical and Computer Engineering, University of Alberta, \\ Edmonton, AB, Canada T6G 2 V4 \\ Correspondence should be addressed to Michael A. Startsev; michael.startsev@mq.edu.au
}

Received 4 January 2013; Accepted 28 January 2013

Academic Editors: X. Chen, A. K. Dharmadhikari, and S. R. Restaino

Copyright (C) 2013 M. A. Startsev and A. Y. Elezzabi. This is an open access article distributed under the Creative Commons Attribution License, which permits unrestricted use, distribution, and reproduction in any medium, provided the original work is properly cited.

\begin{abstract}
Continuous-wave terahertz $(\mathrm{THz})$ radiation spectroscopy was performed on high explosive materials using a tuneable optical parametric oscillator (OPO). Military grade, solid-phase, explosive substances, such as cyclotetramethylenetetranitramine (HMX), cyclotrimethylenetrinitramine (RDX), pentaerythritol tetranitrate (PETN), and composition-4, were spectrally scanned over the 0.7-1.9 THz frequency range under experimental conditions modeling that of "real-world" security screenings. Spectral peak locations and spectral line broadening effects were quantified using a Lorentz lineshape fit algorithm. The full-width half-maximum (FWHM) parameter computed by the Lorentz fit algorithm was shown to help in the identification of samples with broad and sparse spectral characteristics. A concealed explosives identification scheme was demonstrated through raster scan THz frequency radiation imaging at specific OPO tuning frequencies.
\end{abstract}

\section{Introduction}

Terahertz $(\mathrm{THz})$ radiation, typically recognized as the 0.3 to $10 \mathrm{THz}$ frequency region (wavelengths of $1 \mathrm{~mm}$ to $30 \mu \mathrm{m}$ ), offers promising solutions to many of the challenges facing modern security and surveillance systems. $\mathrm{THz}$ radiation can penetrate most common dielectric materials enabling imaging through plastics, clothing, and cardboard. Since metal strongly reflects $\mathrm{THz}$ radiation, concealed metallic weapons can be easily identified via their high contrast shapes and contours within a $\mathrm{THz}$ radiation scanned image. Unlike X-rays, $\mathrm{THz}$ radiation photon energy is low and non-ionizing $(1 \mathrm{THz}=4 \mathrm{meV})$ so there are no adverse health effects associated with prolonged exposure [1].

The allure of $\mathrm{THz}$ radiation technology for security applications also stems from the fact that the collective intermolecular motions and vibrational transitions of materials, such as explosives and illicit drugs, can be probed with $\mathrm{THz}$ frequency radiation [2]. Neither millimeter waves nor infrared (IR) waves have access to these molecular fingerprints. It is envisaged that $\mathrm{THz}$ radiation spectroscopy can be incorporated into a holistic security system that enables noninvasive identification of substances according to their $\mathrm{THz}$ frequency absorption profile while simultaneously imaging packages or persons for concealed weapons.

There have been technological advancements in the development of continuous-wave (CW) $\mathrm{THz}$ radiation sources and detectors. Optically pumped THz Lasers (OPTL) [3], Backward-Wave Oscillators (BWO) [4], Photomixers [5], and Quantum Cascade Lasers (QCL) [6] are capable of efficiently generating $\mathrm{CW} \mathrm{THz}$ radiation while detection of the $\mathrm{CW}$ terahertz radiation can be accomplished with the use of Golay cells [7] and photoacoustic bolometers [8]. However, all $\mathrm{THz}$ generation and detection systems have inherent frequency range limitations. This constraint is particularly detrimental for spectroscopy of solid-phase bulk explosive materials. In a solid-phase explosive substance, the collective molecular motions result in broad continuum spectral features as opposed to their gas-phase counterparts, where isolated molecular motions primary result in sharp spectral absorption features [9]. Thus, it has been a challenge to identify common solid-phase explosives using $\mathrm{THz}$ radiation spectroscopy. 


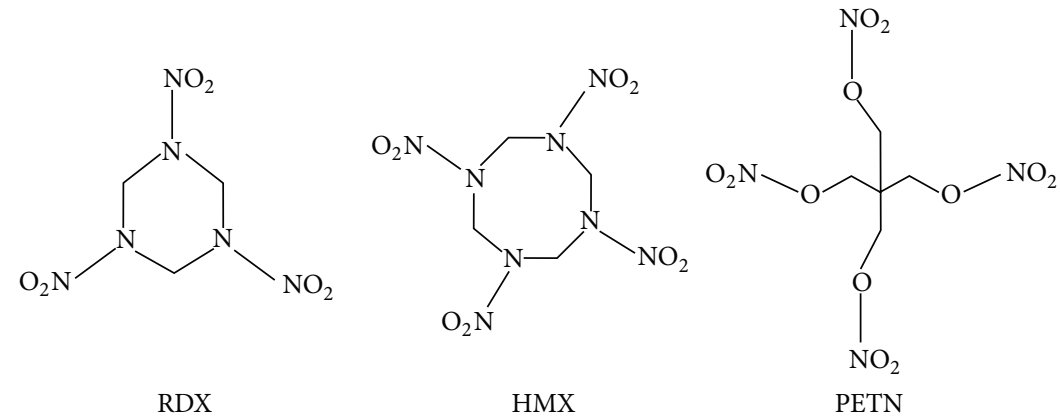

FIGURE 1: Chemical structure diagrams of cyclotrimethylenetrinitramine (RDX), pentaerythritol tetranitrate (PETN), and cyclotetramethylenetetranitramine (HMX). Composition 4 (C-4) explosive is not shown since it is composed of an RDX mixture.

In this investigation, $\mathrm{THz}$ radiation transmission spectroscopy was performed on bulk solid-phase explosives (cyclotetramethylenetetranitramine (HMX), cyclotrimethylenetrinitramine (RDX), pentaerythritol tetranitrate (PETN), and composition 4 (C-4)). The spectroscopy system employed a CW $\mathrm{THz}$ frequency generation source composed of an optical parametric oscillator (OPO) [12] and a Golay cell detector. A Lorentz lineshape fit scheme was presented to help in the quantitative identification of the explosive substances that exhibit sparse, continuum spectral features within the operating frequency range of the spectroscopy system. By observing the coefficients of constituent Lorentz functions, it was possible to distinguish between explosive substances that exhibited seemingly similar spectral characteristics.

\section{Materials and Methods}

2.1. Sample Preparation. Figure 1 illustrates the chemical structure of the cyclotetramethylene-tetranitramine (HMX), cyclotrimethylene-trinitramine (RDX), and pentaerythritol tetranitrate (PETN) samples used. The powder-form explosive samples were weighted at 2-3 grams each, and were contained within standardized $5 \mathrm{~mL}$ volume polystyrene cuvettes (Plastibrand semimicro), and sometimes within small polyethylene plastic bags. The explosives samples were restricted to their pure form (estimated 98\% for incidental impurities). No attempt was made to mix different explosive substances or to dilute the explosive substances that are highly absorbing to $\mathrm{THz}$ radiation because this could potentially affect the volatility of the explosives. Furthermore, this minimal sample manipulation approach avoided many of the spectral peak broadening and spectral peak shift problems associated with the mixing or dilution of explosive samples [13].

2.2. Experimental Methods. The $\mathrm{THz}$ radiation was generated using an optical parametric oscillator (OPO) delivering $10 \mathrm{~nJ}$, $10 \mathrm{~ns} \mathrm{THz}$ pulses at a $50 \mathrm{~Hz}$ repetition rate. The OPO delivers an average $\mathrm{THz}$ radiation output power of $0.5 \mu \mathrm{W}$ and it can be tuned over wide frequency range between $0.7 \mathrm{THz}$ and $1.9 \mathrm{THz}$. The OPO output was focussed using a $10 \mathrm{~cm}$ focal length, $90^{\circ}$ off-axis, $6.35 \mathrm{~cm}$ diameter, and gold coated parabolic mirror. The explosive samples were placed at the focal plane of the parabolic mirror. The Golay cell was situated $1 \mathrm{~cm}$ directly behind the sample in order to capture the maximum amount of the transmitted $\mathrm{THz}$ radiation. In order to mitigate atmospheric absorption of the $\mathrm{THz}$ radiation, the optical path length between the OPO and the sample was kept as short as possible. The OPO's narrow band output frequency was tuned along its entire range of operation (0.7-1.9 THz). The absorption spectrum for each sample was acquired by normalizing the transmission spectrum through the explosive sample to that of the empty sample cell. The absorption data was then processed through a low-pass Butterworth filter in order to improve the spectral plots.

A Lorentz function fit algorithm was utilized to quantify the $\mathrm{THz}$ radiation spectral peaks of the explosives. In the analysis, the Lorentz lineshape function was defined as

$$
L(v)=L_{0}+\frac{2 \beta}{\pi} \frac{\gamma}{4\left(v-v_{0}\right)^{2}+\gamma^{2}},
$$

where $L_{0}$ is the axis offset, $\beta$ is an amplitude multiplicative factor, $v_{0}$ is the center frequency of the function, and $\gamma$ represents the full-width half-maximum (FWHM) of the Lorentz lineshape function. The basic procedure of a Lorentzian fit algorithm involves the superposition of several Lorentzian curves over the measured spectrum data. The algorithm attempts to replicate the curves of the spectrum as accurately as possible by summing the combination of Lorentz lineshape functions. The coefficients of the constituent Lorentz functions, particularly the FWHM and line center, are the key parameters used for explosives identification.

\section{Results and Discussion}

3.1. $\mathrm{THz}$ Spectroscopy. Figure 2 depicts $\mathrm{THz}$ radiation absorption spectroscopy results for RDX, PETN, HMX, and C-4 samples. The spectrum of icing sugar was also included for comparison as a nonexplosive control substance. It should be noted that at the higher region of the THz spectra $(>1.3 \mathrm{THz})$ there is a steady decrease in signal-to-noise ratio due to the lower $\mathrm{THz}$ output power of the OPO at these frequencies. Thus, the slight spectral fluctuations in this region were attributed to noise effects. 


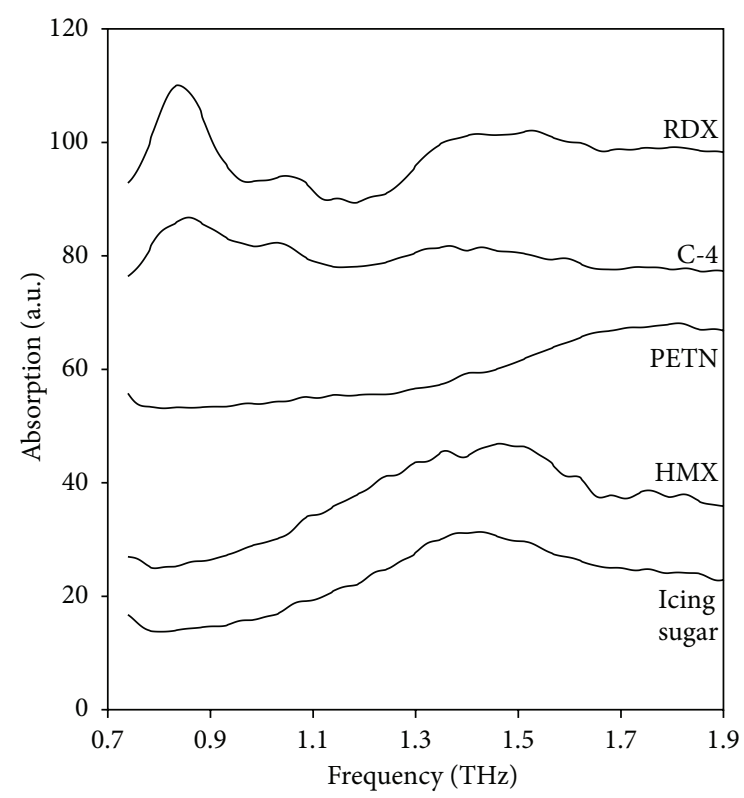

Figure 2: Summary of transmission spectroscopy on explosives. The substances included in order of appearance from the top of the plot are cyclotrimethylene trinitramine (RDX), composition 4 (C-4), pentaerythritol tetranitrate (PETN), cyclotetramethylenetetranitramine (HMX), and icing sugar.

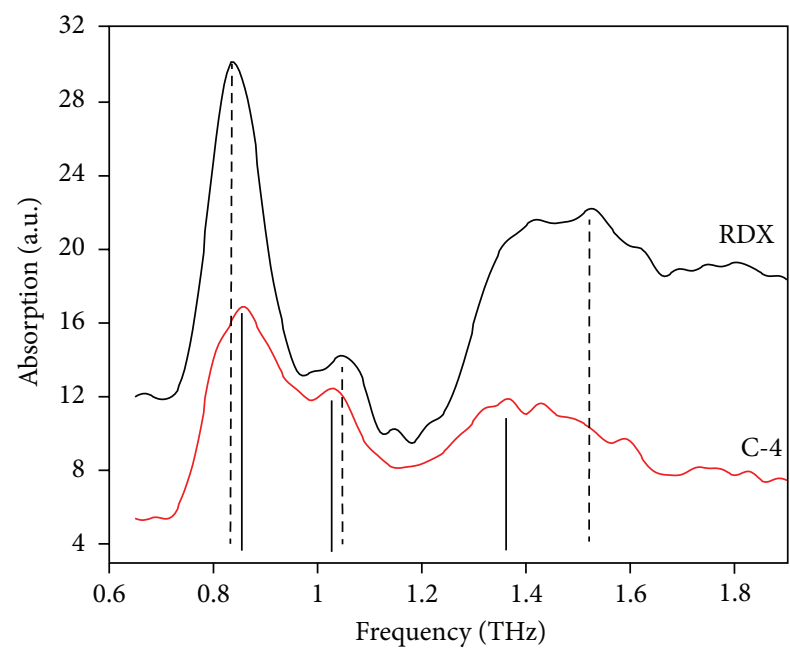

FIgURE 3: Comparison of cyclotrimethylenetrinitramine (RDX) spectrum to the composition $4(\mathrm{C}-4)$ spectrum. RDX spectral peaks are $0.83,1.04,1.51 \mathrm{THz}$ indicated by the vertical dotted lines. C-4 spectral peaks are $0.85,1.02$, and $1.37 \mathrm{THz}$ indicated by the vertical solid lines.

Within the frequency range from $0.7 \mathrm{THz}$ to $1.9 \mathrm{THz}$, both RDX and C-4 exhibit distinct spectral characteristics. A closer examination of the RDX and C-4 spectra shown in Figure 3 illustrates the similarities between the two substances. RDX exhibited absorption peaks of $0.83,1.04$, and $1.51 \mathrm{THz}$ whereas C-4 exhibited peaks of $0.85,1.02$, and 1.37 . The similarities evident between the spectrums were to be

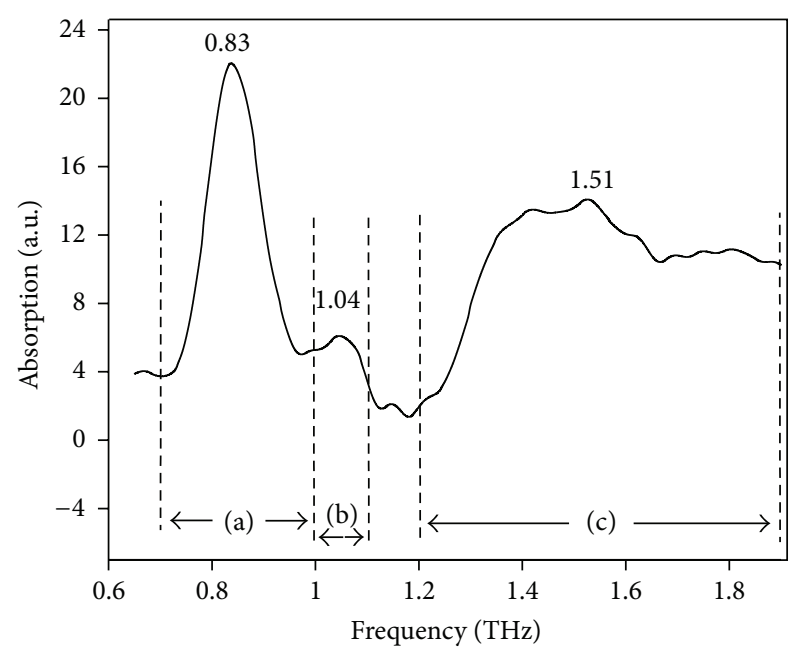

FIGURE 4: Cyclotrimethylene-trinitramine (RDX) THz absorption spectrum. Notations (a), (b), and (c) between the dashed lines represent the segmentation of the plot for Lorentz function analysis shown in Figure 5. Local maxima are annotated at the spectral peaks.

expected since C-4 samples are composed of $91 \%$ RDX, while the remaining $10 \%$ consists of plastic binders, plasticizers, and odorizing taggant chemicals. Notably, both HMX and PETN show nearly featureless absorption spectra, each exhibiting only a single absorption peak within the spectral scan range. In fact, HMX was found to have a similar spectral shape to that of icing sugar. A single broad spectral peak was observed for HMX and it centered about $1.45 \mathrm{THz}$. The center spectral peak of icing sugar was $1.40 \mathrm{THz}$, which appears relatively close to the peak observed for HMX, particularly because both peaks are broad. PETN also exhibited a single spectral peak at $1.78 \mathrm{THz}$ near the upper limit of the frequency tuning range.

In order to correlate the data obtained in this study with previous work, the $\mathrm{THz}$ time domain spectroscopy ( $\mathrm{THz}-$ TDS) studies performed by Liu et al. [10] and Tribe et al. [11] are evoked for comparison. The results in these two studies were in disagreement. Liu et al. found that HMX and PETN explosives have their lowest frequency spectral peaks occurring at 1.78 and $2.0 \mathrm{THz}$, respectively. However, Tribe et al. found these spectral peaks to be 1.58 and $1.73 \mathrm{THz}$, respectively. The THz spectral peaks for HMX and PETN observed in the current study are in closer agreement to the Tribe et al. results.

On the other hand, the RDX spectrum observed in this study is in closer agreement with the results obtained by Liu et al. Our study showed the RDX spectral peaks to be 0.83 , 1.04 , and $1.51 \mathrm{THz}$, which is consistent with the $0.82,1.05$, and $1.50 \mathrm{THz}$ peaks found by Liu et al. Results from the study performed by Tribe et al. found the spectral peaks for RDX to be $0.72,1.26$, and $1.73 \mathrm{THz}$. A summary and comparison of these peaks is presented in Table 1. These discrepancies in spectral peak values suggest that explosives spectra are highly dependent on the specific manufacturing process, which can cause variations in the purity, moisture content, and packing density of explosive powders. 


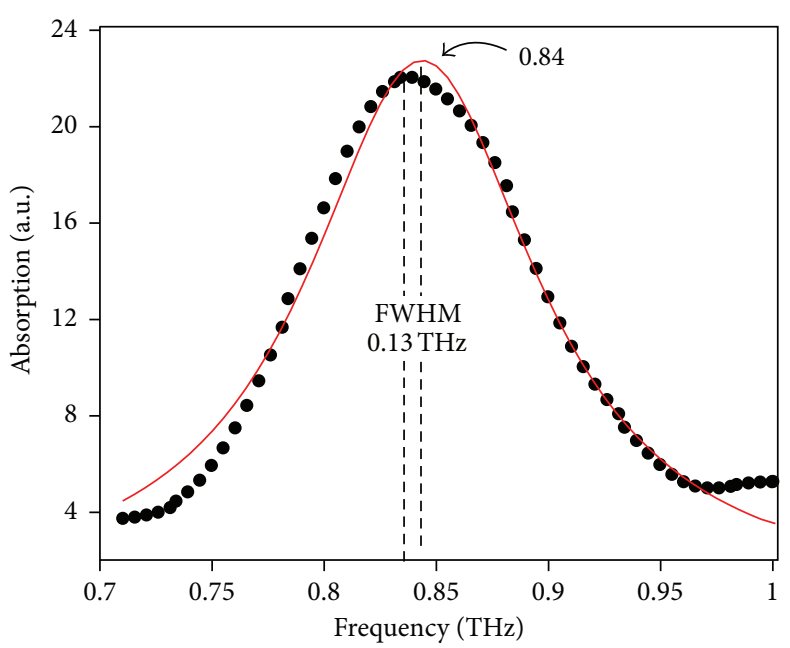

(a)

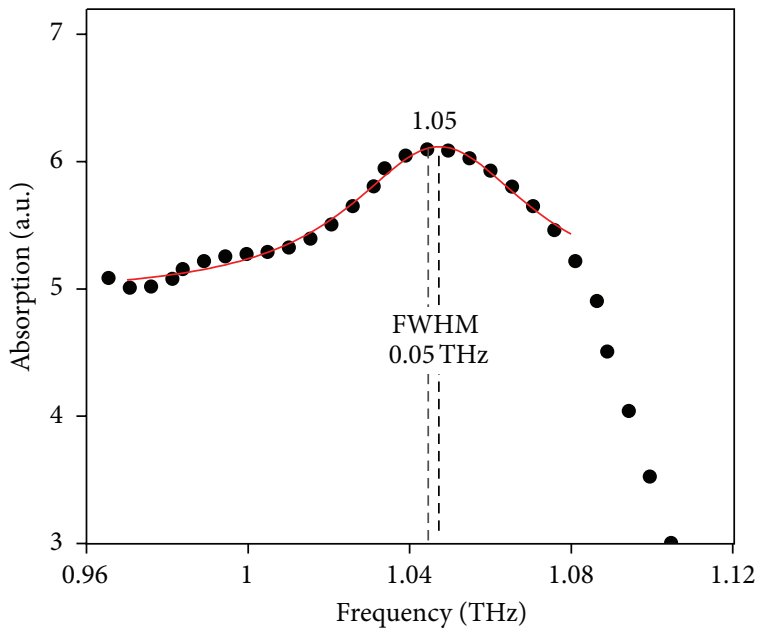

(b)

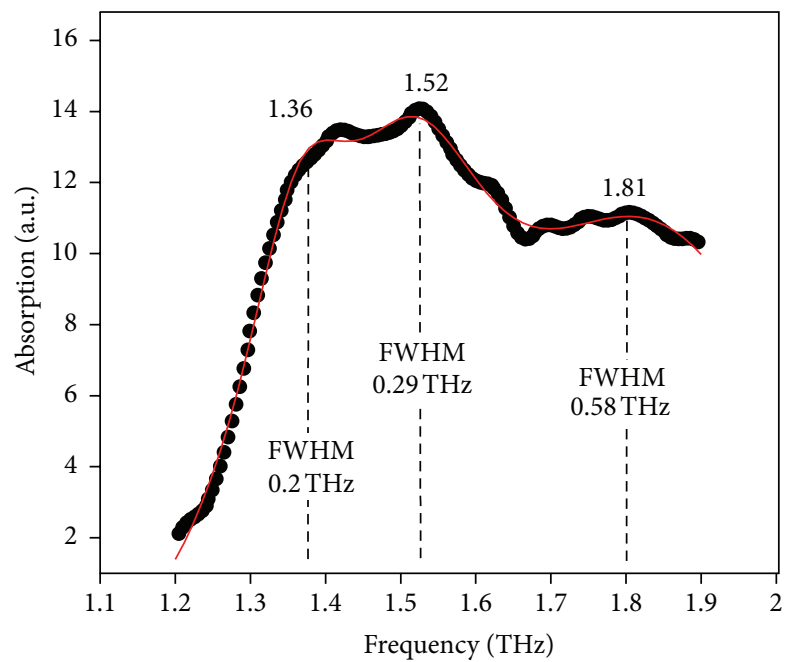

(c)

FIGURE 5: Isolated regions of the cyclotrimethylene-trinitramine (RDX) spectrum with Lorentzian fit analysis. Dots represent the spectrum data points. The solid line represents the Lorentz lineshape function fit. (a) is the $0.7-1.0 \mathrm{THz}$ region, (b) is the $1.0-1.2 \mathrm{THz}$ region, (c) is the 1.2-1.9 THz region as indicated in Figure 4. The plots are annotated with the computed Lorentz fit peaks and their respective full-width half-maximum (FWHM) values.

TABLE 1: Absorption spectral peaks found in spectroscopic experiments performed in this study compared to the peaks that are expected from previous $\mathrm{THz}$ spectroscopic studies [10, 11].

\begin{tabular}{lccc}
\hline $\begin{array}{l}\text { Explosive } \\
\text { compound }\end{array}$ & $\begin{array}{c}\text { Experimental } \\
\text { spectral peaks } \\
(\mathrm{THz})\end{array}$ & $\begin{array}{c}\text { Spectral peaks } \\
\text { from } \\
\text { Lui et al. [10] } \\
(\mathrm{THz})\end{array}$ & $\begin{array}{c}\text { Spectral peaks } \\
\text { from } \\
\text { Tribe et al. [11] } \\
(\mathrm{THz})\end{array}$ \\
\hline RDX & $0.83,1.04,1.51$ & $0.82,1.05,1.50$, & $0.72,1.26,1.73$ \\
HMX & $1.96,2.20,3.08$ & & $1.58,1.91,2.21$, \\
PETN & 1.45 & $1.78,2.51,2.82$ & 2.57 \\
\hline
\end{tabular}

Quantitative analysis was performed on the explosives' spectra using a multipeak Lorentzian fit algorithm. The algorithm derives the spectral peak values and FWHM and obtains the coefficient of determination to assess the accuracy of the fit. Figures 4 and 5 show how the spectral peaks of RDX were separated and analyzed for Lorentz lineshape fit. In Figure 5(a), the Lorentz function indicates the spectral peak to exist at $0.84 \mathrm{THz}$ while the observable local maximum peak is closer to $0.83 \mathrm{THz}$. This slight discrepancy is due to the inherent tendency of the Lorentz fit to conform to an overall symmetry in the spectral peak data. A similar discrepancy was evident for the spectral region shown in Figure 5(b). The local maximum spectral peak was found to be $1.04 \mathrm{THz}$ while the Lorentz fit indicated a $1.05 \mathrm{THz}$ spectral peak. In the 1.21.9 $\mathrm{THz}$ frequency range depicted in Figure 5(c), the Lorentz algorithm most accurately fits the data using three spectral peaks of $1.36,1.52$, and $1.81 \mathrm{THz}$ rather than with a single peak as determined by Liu et al. and Tribe et al. Interestingly, 


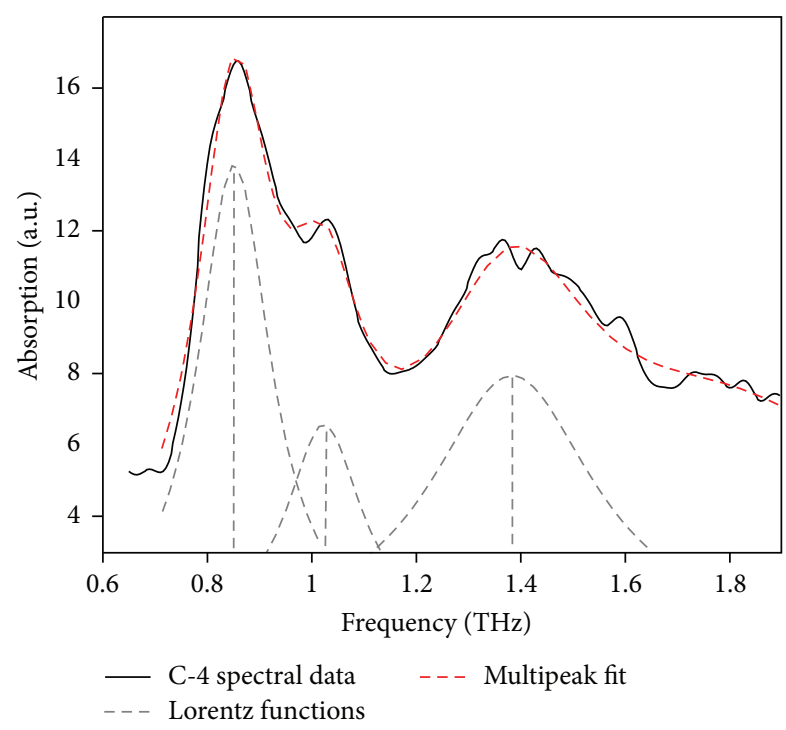

FIgURE 6: Plot of Lorentzian fit algorithm performed on the composition 4 (C-4) absorption spectrum. Grey dashed lines represent individual Lorentz lineshape functions with their central peaks annotated on the plot. The red dashed line represents the superimposed resultant fit. The solid line is the original spectrum. The spectral peaks as computed by the Lorentz algorithm are 0.85 , 1.02 , and $1.38 \mathrm{THz}$.

another study performed by Liu et al. [14], that focussing specifically on the diffuse reflection spectroscopy of RDX, recognized that there is a spectral peak located at the $1.35 \mathrm{THz}$ frequency. Since this spectral peak value is in close agreement to the $1.36 \mathrm{THz}$ spectral peak computed by the Lorentz algorithm in this study, it is conceivable that the multiple peaks computed by the algorithm in the $1.2-1.9 \mathrm{THz}$ region may, indeed, be indicative of "real" molecular resonances. Due to the close proximity of the broadened lineshapes, they are obscured and become difficult to distinguish visually but easy to compute using the Lorentz algorithm.

Similarly, the result of the Lorentz fit computation for C4 is shown in Figure 6. The peaks found by the Lorentz fit closely match the local maxima peaks. Both Lorentz fit and local maximum methods determined the lowest $\mathrm{THz}$ region spectral peaks to be 0.85 and $1.02 \mathrm{THz}$. As opposed to the RDX Lorentz fit, only one Lorentz lineshape function was used to represent the 1.2-1.9 THz spectral region for C-4. The spectral peak in this region was computed by Lorentz fit as a $1.38 \mathrm{THz}$ spectral peak while the local maximum method used earlier showed a $1.37 \mathrm{THz}$ spectral peak. However, as mentioned earlier, the local peak maximum may be affected by the increased noise fluctuation that occurs when the OPO is tuned to higher frequencies. The OPO system exhibited a lower signal-to-noise ratio at higher $\mathrm{THz}$ frequency tunings (i.e., above $1.3 \mathrm{THz}$ ) so the smaller peaks in this region may be attributed to noise fluctuations. The Lorentz algorithm computed peak is more reliable here because it attributes less significance to the noise fluctuations.

The relative shifts in spectral peaks when comparing C4 to RDX, could be attributed to "matrix" effects. Since THz probes the collective intermolecular motions of solid phase explosives, any impurity substances forming a structural matrix, such as plasticizers and binders, could cause changes to the $\mathrm{THz}$ spectrum. The RDX molecules within C-4 interact with the impurity molecules so the collective vibrations are affected and thus the frequency absorptions of C-4 become shifted from that of the pure RDX sample.

The broad spectral signatures of explosives similar to those exhibited by HMX and PETN spectra, shown in Figure 2, could benefit most from the application of the Lorentz fit algorithm. In Figure 7, the utility of the method is apparent by how successfully the spectral peak broadening effect can be quantified using the FWHM coefficient of the generated Lorentz lineshape function. In Figure $7(\mathrm{a})$, the $\mathrm{THz}$ spectrum of HMX is compared to that of icing sugar. The HMX peak determined by the Lorentz function was $1.43 \mathrm{THz}$ which is similar to the Lorentz function spectral peak for icing sugar at $1.44 \mathrm{THz}$. However, the FWHM of HMX was $0.83 \mathrm{THz}$ while icing sugar had FWHM of $0.67 \mathrm{THz}$. Using both, spectral peak and FWHM tabulated values together; HMX can be distinguished from icing sugar. If only local maximum peak values are available, the result would remain inconclusive.

A summary of the Lorentz linefunction fit algorithm results is presented in Table 2. As discussed throughout the paper, some of the spectral peaks found by the Lorentzian algorithm do not precisely match the peaks that were attained by computing the local maxima. However, the Lorentz fit algorithm arguably provides a more reliable assessment of spectra since it takes into consideration the natural symmetry of spectral broadening effects and disregards noise effects. The local maximum method for determining spectral peaks can result in error if noise peak amplitudes exceed the molecular absorption peaks and is, therefore, at a disadvantage for assessing the naturally broad, nearly flat continuum spectrums of solid-phase explosive substances. The Lorentz algorithm computes the fit while considering the general trend of the spectrum in its entirety; it attributes less significance to minor fluctuations and is therefore not as susceptible to noise.

3.2. Continuous-Wave Terahertz Radiation Imaging of Explosives. Imaging and explosives identification can be achieved simultaneously by performing raster scans of an object at multiple $\mathrm{THz}$ frequencies. The image contrast at specific frequencies could help the identify whether a substance within a package is an explosive. As in the spectroscopy trials, the $\mathrm{THz}$ radiation beam was focussed on the target's translation stage using a parabolic mirror. A triangle sample of RDX powder with the dimensions of $1 \times 1 \mathrm{~cm}$ (base $\times$ height) was placed within a paper envelope and attached to the translation stage sample holder. The sample was then imaged at three frequencies: $0.90,0.83$, and $0.77 \mathrm{THz}$ in a raster scan scheme. These three frequencies were chosen because they trace out most distinct characteristic spectral peak of $\mathrm{RDX}$ at $0.83 \mathrm{THz}$ (Figure 4). The results of the images were normalized against an image of an empty envelope scan at each respective frequency. The image results of all three scans are shown in Figure 8. It is evident that, in comparison to 


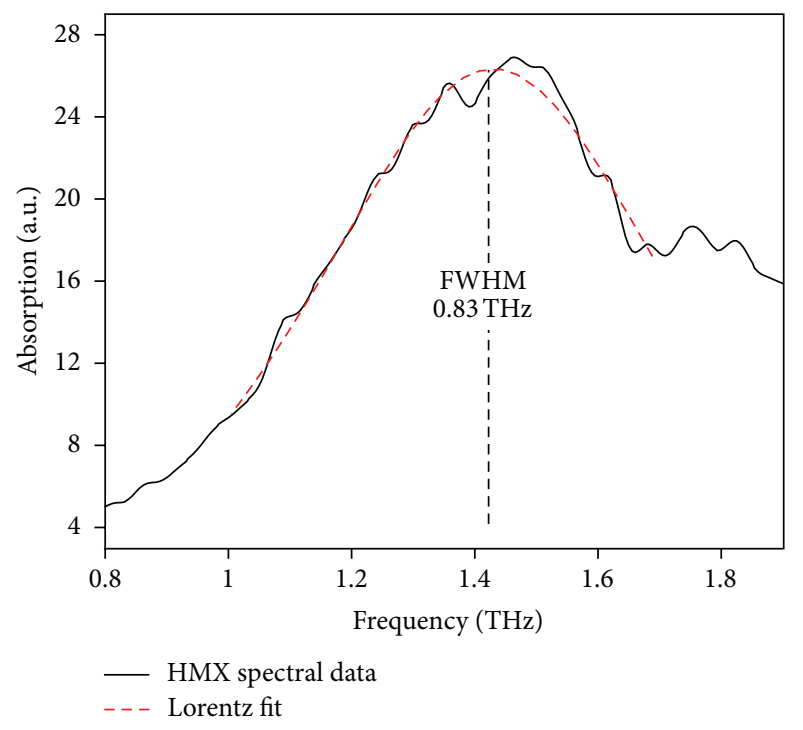

(a)

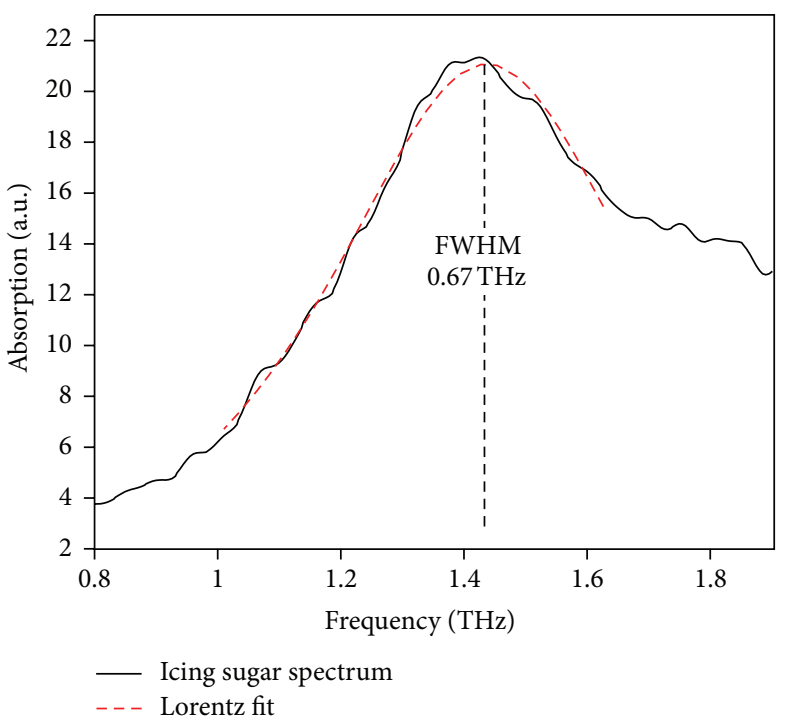

(b)

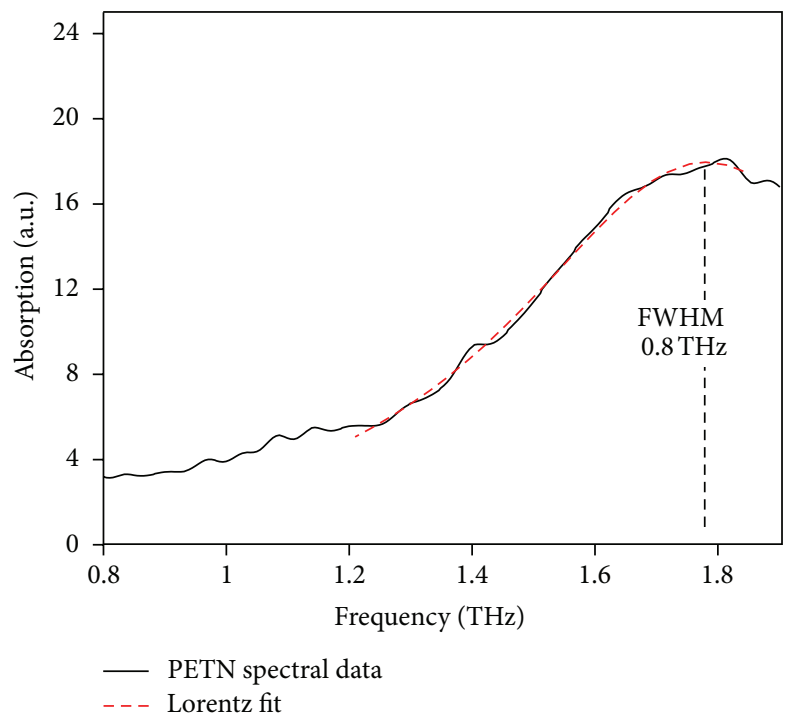

(c)

Figure 7: Absorption curves of (a) cyclotetramethylene-tetranitramine (HMX) compared to (b) icing sugar and (c) pentaerythritol tetranitrate (PETN) with superimposed Lorentz fit functions. The peaks for HMX and icing sugar are similar when found by the Lorentz lineshape function as 1.43 and $1.44 \mathrm{THz}$, respectively. However, the linewidth of HMX is $0.83 \mathrm{THz}$ while icing sugar has a $0.67 \mathrm{THz}$ linewidth as determined by FWHM of the Lorentz function. PETN (c) exhibits a $1.78 \mathrm{THz}$ spectral peak and $0.80 \mathrm{THz}$ FWHM linewidth.

the images attained at $0.77 \mathrm{THz}$ and $0.90 \mathrm{THz}$ OPO tunings, the image contrast at $0.83 \mathrm{THz}$ shows the clearest triangular shape of the RDX explosive. This method essentially allows for simultaneous imaging and spectroscopy using only a few key frequency tunings about an expected spectral peak characteristic to a specific explosive.

\section{Conclusion}

Continuous-wave $\mathrm{THz}$ frequency spectroscopy was performed on powdered explosives. The Lorentz fit algorithm was found to mitigate some of the difficulties associated with conventional means of explosives identification within the limited frequency range of a $\mathrm{THz}$ spectroscopy system. The standard local maxima determination method for identifying spectral peaks was shown to have limited application in the case of HMX and PETN spectral analysis since these substances exhibit broad and sparse spectral characteristics. On the other hand, the Lorentz fit algorithm not only presented an accurate determination of the spectral peaks, but also it provided an additional criterion, the FWHM, with which to determine the spectral signature. While the FWHM estimations within a cluster of spectral peaks may not be easily assessed visually, the Lorentz lineshape function 
TABLE 2: Lorentz fit algorithm peak values and FWHM values for each explosive compared to the spectral peaks obtained by determining the local maximum spectral peak values.

\begin{tabular}{|c|c|c|c|c|}
\hline $\begin{array}{l}\text { Explosive } \\
\text { compound }\end{array}$ & $\begin{array}{c}\text { Local maximum } \\
\text { spectral peaks }(\mathrm{THz})\end{array}$ & $\begin{array}{l}\text { Lorentz function fit of } \\
\text { spectral peaks }(\mathrm{THz})\end{array}$ & FWHM (THz) & $\begin{array}{c}\text { Coefficient of } \\
\text { determination }\left(R^{2}\right)\end{array}$ \\
\hline \multirow{5}{*}{ RDX } & 0.83 & 0.84 & 0.13 & 0.98 \\
\hline & 1.04 & 1.05 & 0.05 & 0.94 \\
\hline & \multirow{3}{*}{1.51} & 1.36 & 0.20 & 0.99 \\
\hline & & 1.52 & 0.29 & 0.99 \\
\hline & & 1.81 & 0.58 & 0.99 \\
\hline HMX & 1.45 & 1.43 & 0.83 & 0.98 \\
\hline Icing sugar & 1.40 & 1.44 & 0.67 & 0.99 \\
\hline \multirow[t]{2}{*}{ PETN } & 1.78 & 1.78 & 0.80 & 0.99 \\
\hline & 0.85 & 0.85 & 0.17 & \\
\hline \multirow[t]{2}{*}{ C- 4} & 1.02 & 1.02 & 0.17 & 0.98 \\
\hline & 1.37 & 1.38 & 0.38 & \\
\hline
\end{tabular}

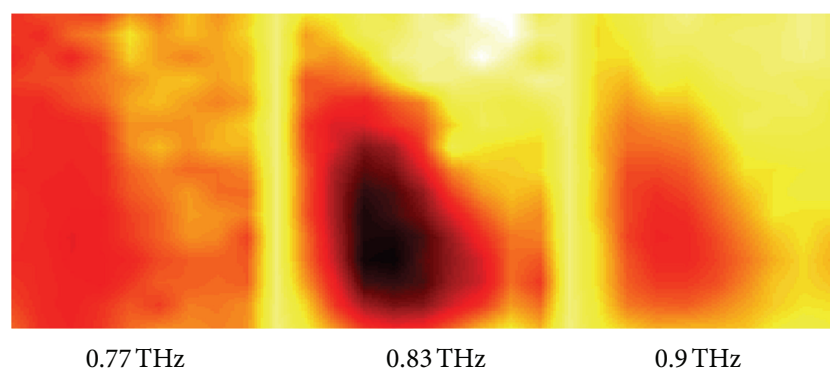

FIGURE 8: Images of a triangle-shaped cyclotrimethylene-trinitramine (RDX) explosive concealed within a paper envelope. Three images are shown using the OPO's tuning frequencies of $0.77,0.83$, and $0.90 \mathrm{THz}$. The highest image absorption contrast is observed at the $0.83 \mathrm{THz}$ tuning frequency.

fit generated these values as coefficients to the constituent Lorentz equations.

For security screening applications, the Lorentz equation coefficients can be referenced to tabulated values and used to assess the chemical compositions of materials. The tabulated Lorentz fit approach to explosives identification can circumvent issues related to identifying explosives that exhibit different spectral characteristics depending on the specific manufacturing process of the sample. The spectral variations of explosives inherent to a specific manufacturing process may be simply added to the Lorentz fit database as an alternative set of parameters to indicate the explosive type. Furthermore, this variation data could reveal the source of the explosive. Eventually, with advancements in technology and techniques, the explosives identification process may be fully automated such that visual inspection of the $\mathrm{THz}$ spectrum would become unnecessary.

The final section of this study demonstrated a security screening application of $\mathrm{CW} \mathrm{THz}$ radiation whereby explosives are selectively screened in conjunction with $\mathrm{THz}$ frequency radiation imaging. Raster scans performed using strategically selected frequencies that traced out a signature spectral peak of RDX resulted in contrast images that indicated the location and shape of RDX powder concealed within an envelope. It is envisaged that a holistic $\mathrm{THz}$ security screening system should combine both quantitative spectral absorption measurements and selective $\mathrm{THz}$ frequency imaging of concealed explosives.

\section{References}

[1] M. R. Scarfi, M. Romanò, R. Di Pietro et al., "THz exposure of whole blood for the study of biological effects on human lymphocytes," Journal of Biological Physics, vol. 29, no. 2-3, pp. 171-177, 2003.

[2] D. L. Woolard, E. R. Brown, M. Pepper, and M. Kemp, "Terahertz frequency sensing and imaging: a time of reckoning future applications?" Proceedings of the IEEE, vol. 93, no. 10, pp. 1722-1743, 2005.

[3] M. Inguscio, G. Moruzzi, K. M. Evenson, and D. A. Jennings, "A review of frequency measurements of optically pumped lasers from 0.1 to $8 \mathrm{THz}$,' Journal of Applied Physics, vol. 60, no. 12, pp. R161-R191, 1986.

[4] M. Mineo and C. Paoloni, "Corrugated rectangular waveguide tunable backward wave oscillator for terahertz applications," IEEE Transactions on Electron Devices, vol. 57, no. 6, pp. 1481$1484,2010$.

[5] E. R. Brown, K. A. McIntosh, K. B. Nichols, and C. L. Dennis, "Photomixing up to $3.8 \mathrm{THz}$ in low-temperature-grown GaAs," Applied Physics Letters, vol. 66, no. 3, pp. 285-287, 1995. 
[6] S. Barbieri, J. Alton, S. S. Dhillon et al., "Continuous-wave operation of terahertz quantum-cascade lasers," IEEE Journal of Quantum Electronics, vol. 39, no. 4, pp. 586-591, 2003.

[7] M. J. E. Golay, "Theoretical consideration in heat and infra-red detection, with particular reference to the pneumatic detector," Review of Scientific Instruments, vol. 18, no. 5, pp. 347-356, 1947.

[8] P. L. Richards, "Bolometers for infrared and millimeter waves," Journal of Applied Physics, vol. 76, no. 1, pp. 1-24, 1994.

[9] D. Mittleman, Sensing with Terahertz Radiation, pp. 40-43, Springer, GmbH \& Co. KG, Berlin, Germany, 2010.

[10] H. B. Liu, H. Zhong, N. Karpowicz, Y. Chen, and X. C. Zhang, "Terahertz spectroscopy and imaging for defense and security applications," Proceedings of the IEEE, vol. 95, no. 8, pp. 15141527, 2007.

[11] R. W. Tribe, A. D. Newnham, F. P. Taday, and C. M. Kemp, "Hidden object detection: security applications of terahertz technology," Proceedings of the Society of Photo Optical Instrumentation Engineers, vol. 5354, pp. 168-176, 2004.

[12] J. I. Shikata, K. Kawase, K. I. Karino, T. Taniuchi, and H. Ito, "Tunable terahertz-wave parametric oscillators using $\mathrm{LiNbO}_{3}$ and $\mathrm{MgO}: \mathrm{LiNbO}_{3}$ crystals," IEEE Transactions on Microwave Theory and Techniques, vol. 48, no. 4, pp. 653-661, 2000.

[13] J. F. Federici, B. Schulkin, F. Huang et al., “THz imaging and sensing for security applications-explosives, weapons and drugs," Semiconductor Science and Technology, vol. 20, no. 7, pp. S266-S280, 2005.

[14] H. B. Liu, Y. Chen, G. J. Bastiaans, and X. C. Zhang, "Detection and identification of explosive RDX by $\mathrm{THz}$ diffuse reflection spectroscopy," Optics Express, vol. 14, no. 1, pp. 415-423, 2006. 

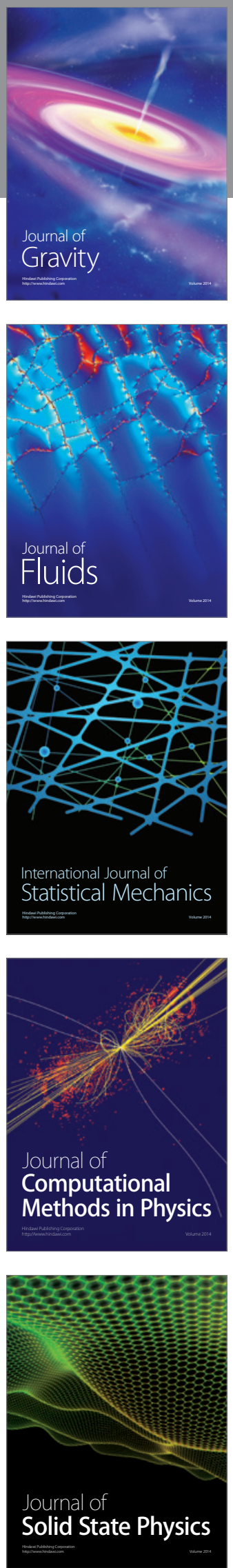

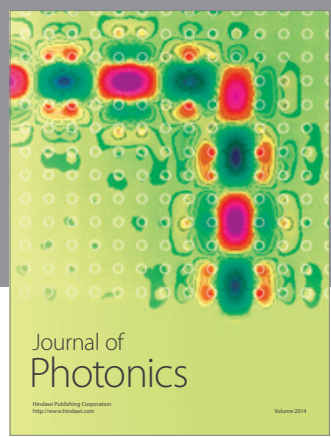

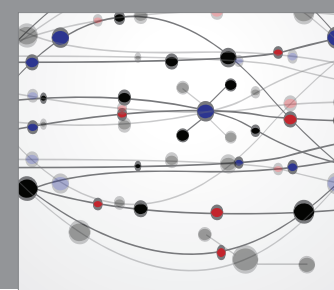

The Scientific World Journal

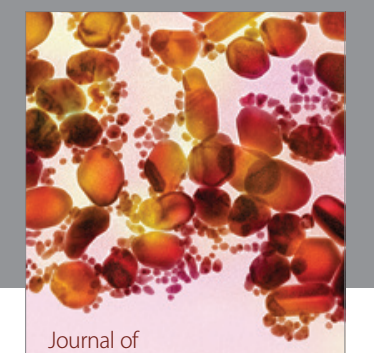

Soft Matter
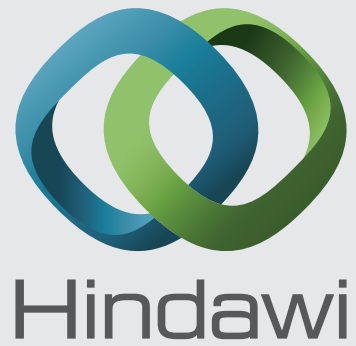

Submit your manuscripts at

http://www.hindawi.com
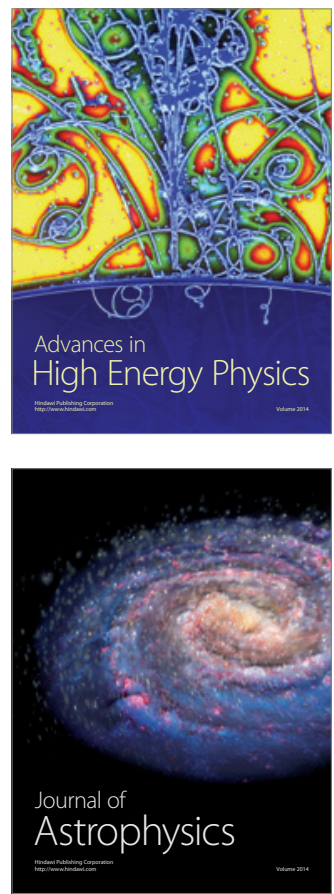
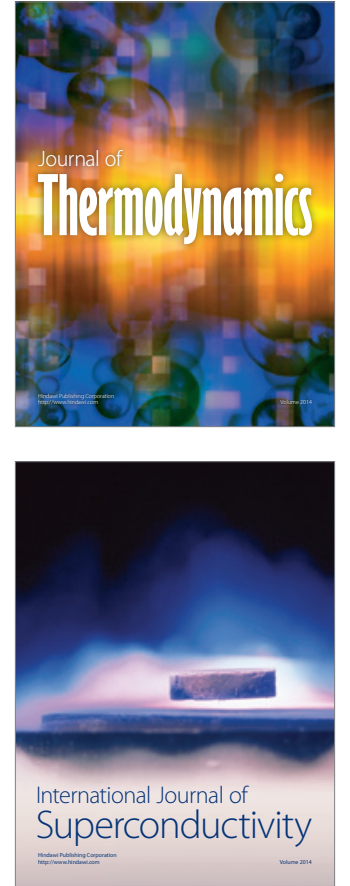
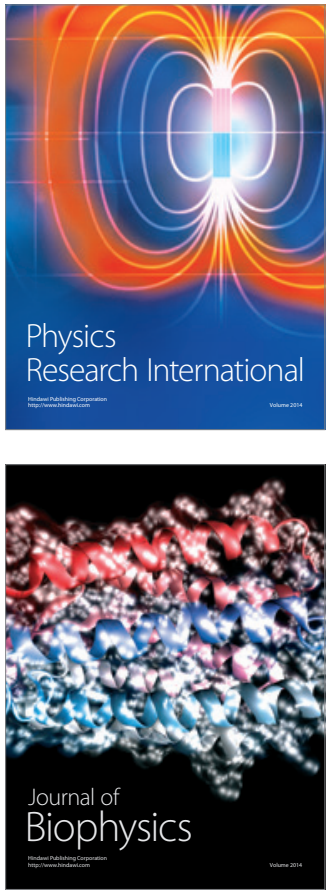
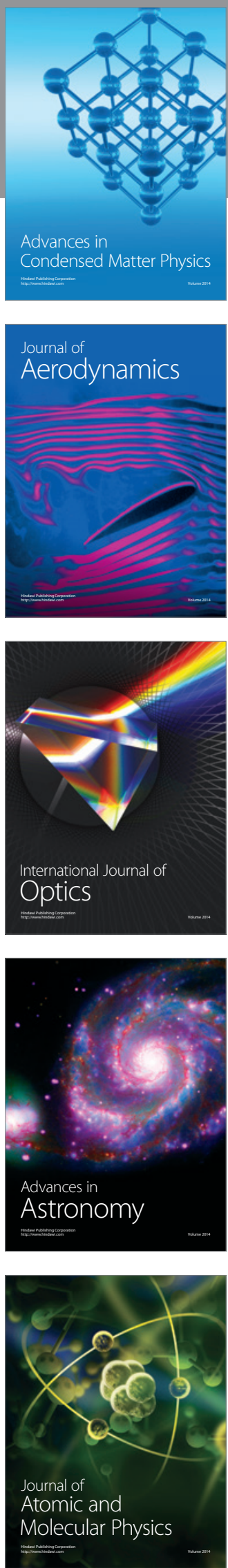